\section{A SINGLE-CELL SPATIALLY RESOLVED MERFISH MAP OF THE COLORECTAL TUMOR IMMUNE MICROENVIRONMENT}

${ }^{1}$ Colles Price* ${ }^{2}$ Jonathan Chen, ${ }^{3}$ Karin Pelka, ${ }^{4}$ Sherry Chao, ${ }^{1}$ Jiang He, ${ }^{5}$ Genevieve Boland, ${ }^{1}$ George Emanuel, ${ }^{6}$ Nir Hacohen. 'Vizgen, Cambridge, MA, United States; ${ }^{2} M G H$, Cambridgee, MA, United States; ${ }^{3}$ Broad Institute, Cambridge, MA, United States; ${ }^{4}$ Harvard University, Cambridge, MA, United States; ${ }^{5}$ Harvard Medical School, MGH, Cambridge, MA, United States; ${ }^{6}$ MGH, Harvard Medical School, Cambridge, MA, United States

Background Understanding the tumor microenvironment (TIME) requires more than just a catalog of cell types and gene programs. It is critical to see the spatial organization of the cells are and where they form multicellular interaction networks. Here we present a single-cell spatially resolved transcriptomic analysis of human mismatch repair deficient (MMRd) and proficient (MMRp) colorectal cancer (CRC) specimens. High tumor mutational burden MMRd tumors are known to have an immune response characterized by higher cytolytic $\mathrm{T}$ cell infiltrates compared to MMRp tumors, making them an ideal system for spatial single-cell profiling and understanding how the immune-driven programs differ between these tumors.

Methods MERFISH is a massively multiplexed single molecule imaging technology which can simultaneously capture and measure the quantity and distribution of hundreds to thousands of RNA species within single cells across a tissue. ${ }^{1}$ We designed a MERFISH library of over 450 genes including genes important to proliferation, apoptosis, immune signaling, immune cell type pathways and other critical pathways in CRC. Patient samples were obtained commercially or through Massachusetts General Hospital. Samples were hybridized with the designed MERFISH library and stained with a cell boundary marker to delineate cells across the tissue. We performed unsupervised clustering to identify cell types and we explored calculated spatial statistics to characterize how the cell type distribution varied between MMRd and MMRp tumors. We identified the cellular composition of each tumor, including immune and stromal cells, and the spatial distribution of these cell types.

Results Using MERFISH, we were able to readily identify all cell types and states previously discovered by single-cell RNA sequencing $^{2}$ in intact patient specimens, thus providing an accurate map of the cellular composition and spatial organization of these cells in the tumor microenvironment. Of note, previously predicted multicellular interaction networks 2 appeared as spatially organized structures in the tissue and were distinct in MMRd versus MMRp tumor specimens. Our data provide a richness of concrete hypotheses about which cells are working together and how these cells function cooperatively, which will be critical in advancing immunotherapy in these immunologically distinct types of colorectal cancer.

Conclusions Here we present a single-cell resolved spatial map of the cell types and states in the tumor microenvironment of MMRd and MMRp cancer. This will aid the development of future immunotherapies for CRC patients.

\section{REFERENCES}

1. Chen KH, Boettiger AN, Moffitt JR, Wang $S$, Zhuang X. RNA imaging. Spatially resolved, highly multiplexed RNA profiling in single cells. Science 2015;348:AAA 6090.

2. Pelka K, Hofree M, Chen J, Sarkizova S, Pirl JD, Jorgji V, et al. Multicellular immune hubs and their organization in MMRd and MMRp colorectal cancer. BioRxiv 2021;426796.
Ethics Approval All samples not commercially purchased were collected in accordance with IRB protocol DF/HCC IRB 02 240.

http://dx.doi.org/10.1136/jitc-2021-SITC2021.920 